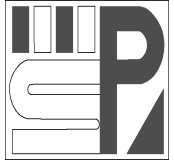

Science Press

Journal of Arid Land

2012, 4(4): 390-398

doi: 10.3724/SP.J.1227.2012.00390

jal.xjegi.com; www.chinasciencejournal.com

\title{
A simulation-based two-stage interval-stochastic programming model for water resources management in Kaidu-Konqi watershed, China
}

\author{
Yue HUANG ${ }^{1,2}$, Xi CHEN ${ }^{2 *}$, YongPing $\mathrm{LI}^{3}$, AnMing BAO${ }^{2}$, YongGang MA ${ }^{2,4}$ \\ ${ }^{1}$ College of Urban and Environmental Sciences, Peking University, Beijing 100871, China; \\ ${ }^{2}$ State Key Laboratory of Desert and Oasis Ecology, Xinjiang Institute of Ecology and Geography, Chinese Academy of \\ Sciences, Urumqi 830011, China; \\ ${ }^{3}$ MOE Key Laboratory of Regional Energy Systems Optimization, Sino-Canada Resources and Environmental Research \\ Academy, North China Electric Power University, Beijing 102206, China; \\ ${ }^{4}$ Xinjiang Remote Sensing Center, Urumqi 830011, China
}

\begin{abstract}
This study presented a simulation-based two-stage interval-stochastic programming (STIP) model to support water resources management in the Kaidu-Konqi watershed in Northwest China. The modeling system coupled a distributed hydrological model with an interval two-stage stochastic programing (ITSP). The distributed hydrological model was used for establishing a rainfall-runoff forecast system, while random parameters were provided by the statistical analysis of simulation outcomes. The developed STIP model was applied to a real case of water resources management planning in Kaidu-Konqi watershed, where three scenarios with different water resources management policies were analyzed. The results indicated that water shortage mainly occurred in agriculture, ecology and forestry sectors. In comparison, the water demand from municipality, industry and stockbreeding sectors can be satisfied due to their lower consumptions and higher economic values. Different policies for ecological water allocation can result in varied system benefits, and can help to identify desired water allocation plans with a maximum economic benefit and a minimum risk of system disruption under uncertainty.
\end{abstract}

Keywords: optimization; two-stage stochastic programming; uncertainty; water resources management; hydrological model; Kaidu-Konqi watershed; Tarim River Basin

In arid and semiarid regions, water resources management has become an increasingly pressing issue (Paturel et al., 2007). Most inland river basins in such regions suffer markedly ecological degradations and vegetation losses (Bronstert et al., 2000; Li et al., 2006; Yang et al., 2010; Jing and Chen, 2011; Chen et al., 2012). These issues have forced planners to propose more comprehensive, complex and ambitious plans for water resource management systems (Li et al., 2006). Moreover, spatial and temporal variations can exist in many system components (e.g. stream flows, water demands, and water-allocation targets) and fluctuations can be associated with net system benefits, which are functions of many stochastic factors. These complexities could become further compounded by not only the interactions among uncertain parameters but also their economic implications. As a result, the inherent complexities and stochastic uncertainties that exist in real-world water resource management systems have essentially placed them beyond the conventional deterministic optimization methods (Qin et al., 2009).

Two-stage stochastic programming (TSP) method is an effective alternative for tackling problems where an analysis of policy scenarios is desired and the related data are mostly uncertain (Huang and Loucks, 2000; Maqsood et al., 2005). In TSP, a decision is first un-

Received 2012-02-09; accepted 2012-06-01

"Corresponding author: Xi CHEN (E-mail: chenxi@ms.xjb.ac.cn) 
dertaken before values of random variables are known and, then, after the random events have happened and their values are disclosed, a second decision is made minimize "penalties" that may appear due to any infeasibility. In the past decades, many studies dealt with uncertainties presented in multiple formats by integrating interval-parameter programming (IPP) and/or fuzzy programming (FP) methods into the TSP framework (Li et al., 2006). For example, Huang and Loucks (2000) proposed an inexact two-stage stochastic programming (ITSP) model to address uncertainties expressed as probability density functions (PDFs) and discrete intervals. Luo et al. (2006) presented a simulation-based ITSP model for agricultural nonpoint source pollution control caused by land retirement under uncertain conditions. Li and Huang (2008) developed an interval-parameter two-stage stochastic nonlinear programming method for supporting water resource-allocation decisions for a multi-reservoir system. Li et al. (2010) proposed an inexact two-stage water management model for agricultural irrigation in the Zhangweinan River Basin of China. However, few researchers have focused on water resources allocation and optimization under uncertainty by applying these stochastic-based models in arid and semi-arid watersheds.

This study aims to extend previous efforts by developing a simulation-based two-stage interval-stochastic programming (STIP) model for water resources management planning. The developed STIP can be applied to planning water resources allocation in the Tarim River Basin, a region plagued by severe water shortages and an extremely vulnerable ecological system. Due to vegetation degradation and soil erosion, the conflict between ecological protection and economic development has become increasingly extrusive in the exploitation and utilization of water resources; the sustainable development of the regional society and economy has also been seriously restricted (Huang et al., 2010a). The results can help decision makers establish effective water exploitation and allocation policies to enhance the sustainability of the local ecosystems.

\section{Methods}

\subsection{Hydrological modeling}

Prediction of the volume of available water resources is the basis for water resources planning and management, and hydrological models are effective tools for resolving this problem (Bronster, 2000; Wang et al., 2011). MIKE SHE model is a physically-based distributed hydrological modeling system covering the entire land phase of the hydrological cycle (Abbott et al., 1986). It consists of five modules: overland flow, evapotranspiration, unsaturated flow, saturated flow, and channel flow modules (DHI, 2007). In this study, a rainfall-runoff model based on MIKE SHE modeling system was used to simulate stream flows. Overland flow was based on the dynamic solution of the two-dimensional Saint-Venant equations. Channel flow was calculated using a one-dimensional simulation produced by the MIKE 11 Hydrodynamic Module (DHI, 2007), and a finite difference approach and double sweep algorithm were used to solve the unsteady flow equations.

\subsection{STIP model}

The STIP model was developed by coupling the MIKE SHE model with the ITSP. Water allocation of an entire system should be reassessed to maximize its mean annual net benefit (only annual model is considered without loss of generality). This study considers an arid watershed system where water is released for ecological use. If the promised amount of water is delivered, a net benefit to the local economy will be generated for each unit of water allocated (denoted as $\left.B_{i}\right)$. However, if the promised amount of water cannot be delivered, the user will have to either obtain water from more expensive alternatives or curb their development plans. Associated penalties (denoted as $\mathrm{C}_{\mathrm{i}}$ ) are the acquisition cost of water from expensive alternatives and the cost of negative consequences generated from the curbing of the development plans (Li et al., 2008). Uncertainties exist not only in water availability, but also in economic parameters. However, the quality of information that can be obtained for these uncertainties is mostly not satisfactory enough to be 
presented as probability distributions. Thus, interval parameters are introduced into the optimization framework (Huang and Cao, 2011). The problem of water allocation can be analyzed by formulating an interval two-stage stochastic programming (ITSP) model as follows (Huang and Loucks, 2000):

$$
\operatorname{Max} f^{ \pm}=\sum_{i=1}^{m} B_{i}^{ \pm} W_{i}^{ \pm}-\sum_{i=1}^{m} \sum_{j=1}^{n} p_{j} C_{i}^{ \pm} S_{i j}^{ \pm}
$$

subject to:

$$
\begin{gathered}
\sum_{i=1}^{m}\left(W_{i}^{ \pm}-S_{i j}^{ \pm}\right) \leq q_{j}^{ \pm}, \forall i, \\
S_{i j}^{ \pm} \leq W_{I}^{ \pm} \leq W_{i \max }^{ \pm}, \forall i, \\
S_{i j}^{ \pm} \geq 0, \forall i, j .
\end{gathered}
$$

Where $f^{ \pm}$is net system benefit; $B_{i}^{ \pm}$is the net benefit for user $i$ per unit of water allocated; $W_{i}^{\star}$ is the targeted water allocation that is promised to user $i$; $C_{I}^{ \pm}$is loss to user $i$ per unit of water not delivered, $C_{i}>B_{i} ; S_{i j}^{\sharp}$ is the amount by which water allocation target $W_{i}^{\star}$ is not met when the seasonal flow is $q_{j}^{ \pm}$; $W_{\text {imax }}^{ \pm}$is the maximum allowable allocation amount for user $i ; q_{j}^{ \pm}$is the amount of seasonal flow under level $j ; p_{j}$ is the prob-

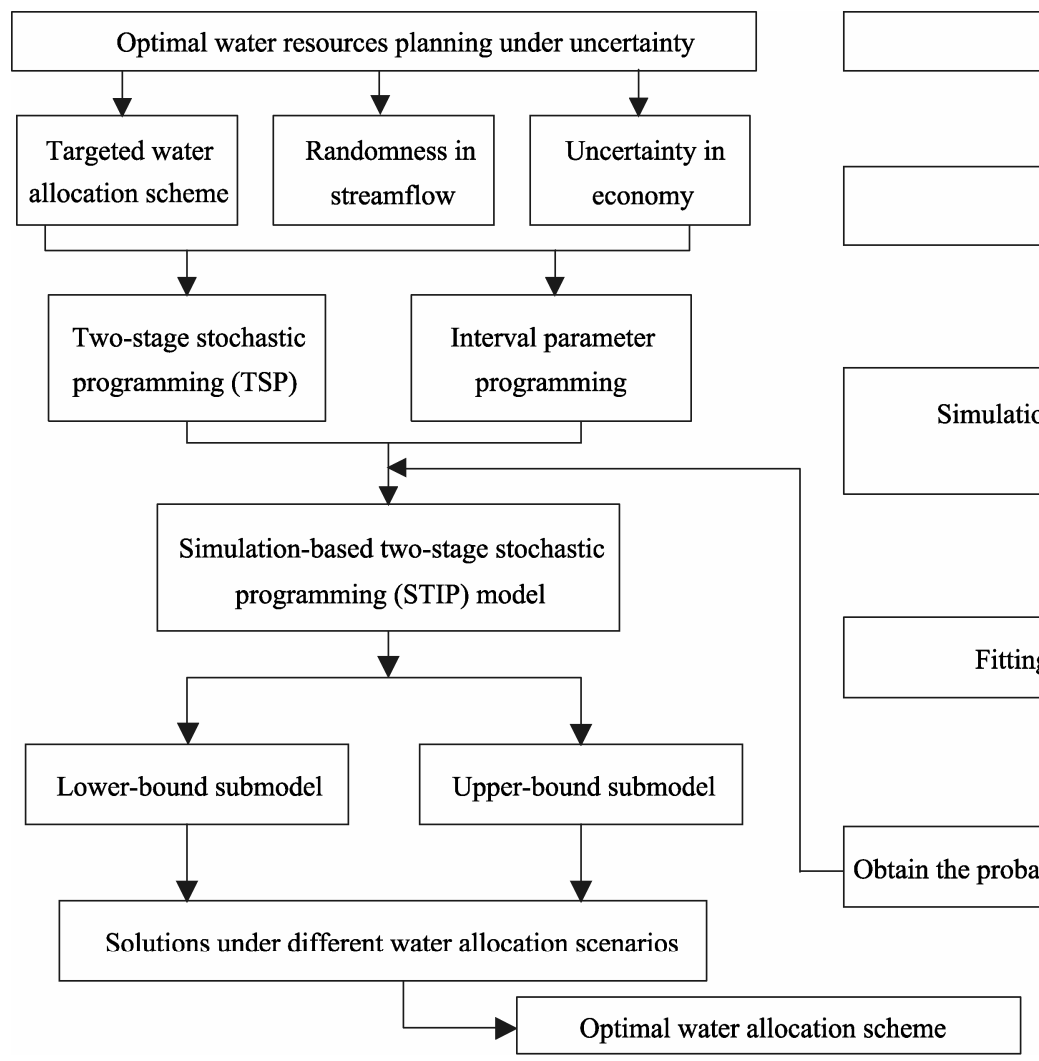

Fig. 1 Schematic of the STIP model ability of the occurrence of flow level $j ; n$ is the total number of flow levels; $m$ is the total number of water users; $i$ is water user, with $i=1$ for municipality, $i=2$ for industry, $i=3$ for stockbreeding, $i=4$ for forestry, $i=5$ for agriculture and $i=6$ for ecology; $j$ is flow level, with $j=1$ representing low flow, $j=2$ for low-medium flow, $j=3$ for medium flow, $j=4$ for medium-high flow, and $j=5$ for high flow.

Figure 1 illustrates the general framework of the STIP model, which is based on the distributed stream flow model and ITSP method (Luo et al., 2006). The solution algorithm of model (1) can then be summarized by using the following pseudo-code:

Step 1: Calibrate and validate the hydrological components of the MIKE SHE model;

Step 2: Use the verified MIKE SHE model to generate the annual stream flow time series (i.e. $q_{j}^{ \pm}$) if over 35 years;

Step 3: Fit the PDFs to obtain the discretization values of $q_{j}^{ \pm}$, and then calculate their probability levels under given $q_{j}^{ \pm}$interval values;

Step 4: Formulate the ITSP model;

Step 5: Solve the model and obtain its solutions.

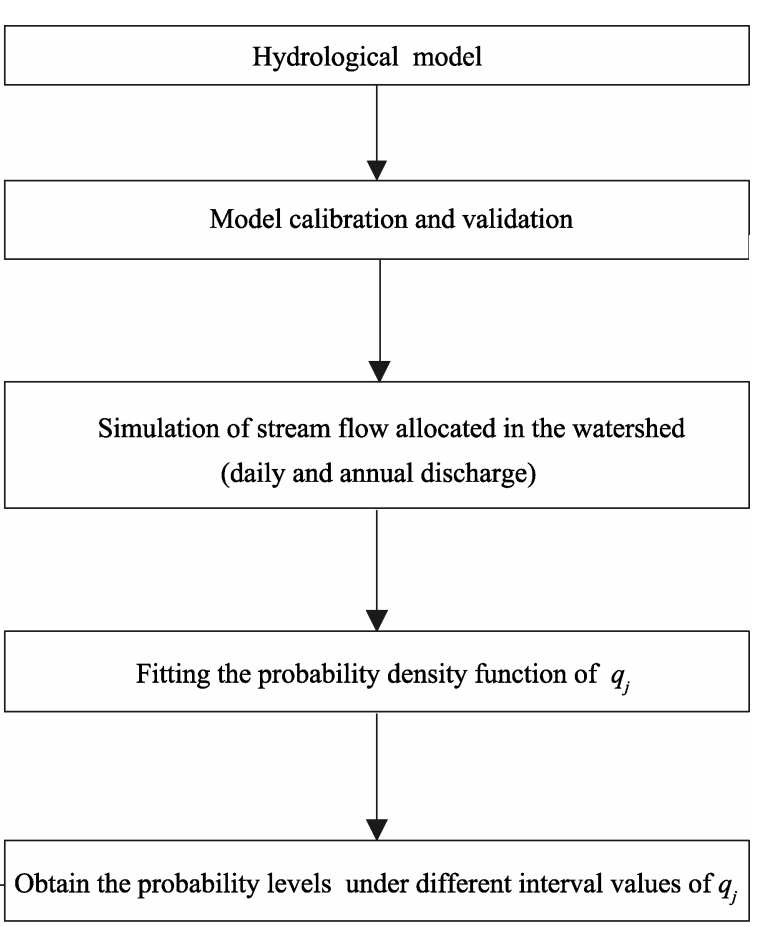




\section{Case study}

\subsection{Study area}

The Kaidu-Konqi watershed is located in the Bayangol Mongol autonomous prefecture in Xinjiang Uygur autonomous region of China. It is one of the four mainstreams of the Tarim River, and has an area of approximately $31.4 \times 10^{3} \mathrm{~km}^{2}$. There are three hydrological stations, i.e. Bayanbulak Station (BYBLK), Dashankou Station (DSK) and Tashidian Station (TSD), along the Kaidu-Konqi River. The observed discharge data from the DSK were used for calibration and validation of the MIKE SHE model. Figure 2 shows the outline of the watershed. The ecosystems in this region are extremely vulnerable to disturbances and habitat degradation due to a severe lack of water resources (Xu, 2007; Huang et al., 2010a). The intensified confrontation between environmental protection and economic development in the region has been inevitable as a result of exploitation and utilization of the limited water resources for local economic activities. Poor management practices have led to severe environmental degradation in the early stages of land reclamation, and the problems would be further exacerbated by continued deforestation and irrigation-induced salinity ( $\mathrm{Xu}$ et al., 2007). Sustainable development of the regional society and economy, therefore, has been seriously constrained. In order to recover the ecological system in the lower reaches of the watershed, local planners have been conducting ecological water releases from the KaiduKonqi River every year since 2000 .

Water from upstream of the Kaidu-Konqi River is allocated for six usages: municipality, industry, stockbreeding, forestry, agriculture and ecology. Competition for water exists among different users when available water cannot satisfy demands. All users want to know how much water they can expect for the next year. The simulation-based optimization model could be used for allocating and managing water in more efficient and environmentally friendly ways (Huang et al., 2010b). Therefore, it is deemed necessary to develop an effective simulation-optimization system for supporting water resources management in the study area under such conditions (Li et al., 2008).

\subsection{Data collection}

Meteorological data including air temperature, pan evaporation and daily rainfall were collected. Initial condition parameters could be defined in the MIKE SHE model according to references from the Danish Hydraulic Institute (DHI, 2007). Land cover was classified according to a Landsat TM image of 2004 (Liu et al., 2008). The soil map was obtained from a MAPGIS-based database. The input topography map, river network and boundaries of the watershed were derived from the $90 \mathrm{~m} \times 90 \mathrm{~m}$ digital elevation model (DEM). Main parameter values for different water users in model (1) are shown in Table 1, such as

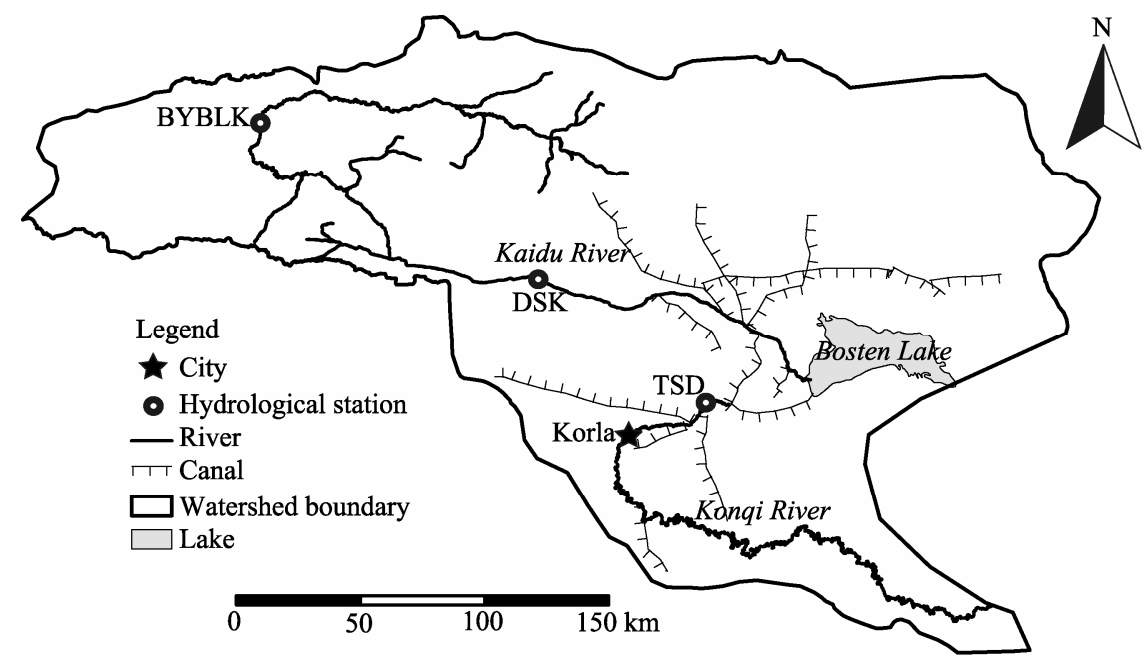

Fig. 2 The map of Kaidu-Konqi watershed 
Table 1 Allowable water allocation and the related economic data under scenario 1

\begin{tabular}{|c|c|c|c|c|c|c|}
\hline Parameter & Municipality & Industry & Stockbreeding & Forestry & Agriculture & Ecology \\
\hline Maximum allowable allocation $\left(W_{\text {imax }}^{ \pm}, 10^{6} \mathrm{~m}^{3}\right)$ & 200 & 900 & 200 & 600 & 3,500 & 1,200 \\
\hline Water allocation target $\left(W_{i}^{ \pm}, 10^{6} \mathrm{~m}^{3}\right)$ & {$[69,191]$} & {$[469,803]$} & {$[41,155]$} & {$[407,527]$} & {$[2,335,3,113]$} & {$[445,1,051]$} \\
\hline $\begin{array}{l}\text { Net benefit when water demand is satisfied } \\
\left(B_{i}^{ \pm}, \$ / 10^{3} \mathrm{~m}^{3}\right)\end{array}$ & {$[7,857,10,000]$} & {$[1,820,6,414]$} & {$[3,900,6,485]$} & {$[257,371]$} & {$[341,455]$} & {$[157,429]$} \\
\hline $\begin{array}{l}\text { Reduction of net benefit when demand is not satisfied } \\
\left(C_{i}^{ \pm}, \$ / 10^{3} \mathrm{~m}^{3}\right)\end{array}$ & {$[14,285,18,571]$} & {$[3,600,11,442]$} & {$[7,857,12,857]$} & {$[515,742]$} & {$[642,1,000]$} & {$[285,643]$} \\
\hline
\end{tabular}

the maximum allowable allocation $\left(W_{\text {imax }}^{ \pm}\right)$, water allocation target $\left(W_{i}^{\star}\right)$, net benefit when water demand was satisfied $\left(B_{i}^{ \pm}\right)$and reduction of net benefit when promised water was not delivered $\left(C_{i}^{ \pm}\right)$. These values can be obtained from the statistical yearbook or derived from published reports and papers (Statistical Bureau of Bayangol Mongol Autonomous Prefecture, 2000-2009; Li et al., 2008). For example, water demand parameters (i.e. $W_{\text {imax }}^{+}$and $W_{i}^{t}$ ) were estimated by local statistical data, and the economical parame ters (i.e. $B_{i}^{ \pm}$and $C_{i}^{\ddagger}$ ) for each water user were derived from related references ( $\mathrm{Li}$ et al., 2006). In this study,three scenarios were considered: (i) scenario 1 was based on current water resources allocation policies (defined as $\mathrm{S}_{1}$ ); (ii) scenario 2 considered ecological issues first. Thus, the priority was given to the ecology sector, whose required minimum demand (i.e. $100 \times 10^{6} \mathrm{~m}^{3}$ ) was satisfied first (denoted as $\mathrm{S}_{2}$ ); and (iii) scenario 3 pursued an aggressive ecological water release policy, in which $60 \%$ of required demand from the ecology sector was satisfied (denoted as $\mathrm{S}_{3}$ ).

\subsection{Modeling approach}

To get the optimal water allocation schemes for the studied watershed, the values of $q_{j}^{ \pm}$should be obtained first. These values can be acquired through statistical analyses with annual stream flow simulation results of the Kaidu-Konqi River which supplies water to the watershed. Figure 3 shows predicted and observed daily runoffs at DSK. The predicted data corresponded to the observed data with a Nash-Sutcliffe coefficient (i.e. $\mathrm{R}^{2}$ ) (Nash and Sutcliffe, 1970) value of 0.79 and 0.71 at the DSK station during the calibration and validation periods, respectively. From the simulation results, the annual discharge during 1970-2005 was calculated. The Gamma distribution was used to fit the
PDFs of random variables (Luo et al., 2006). After the PDFs of the random variable $q_{j}$ were determined, the discretization values with different probability levels were calculated (Table 2).
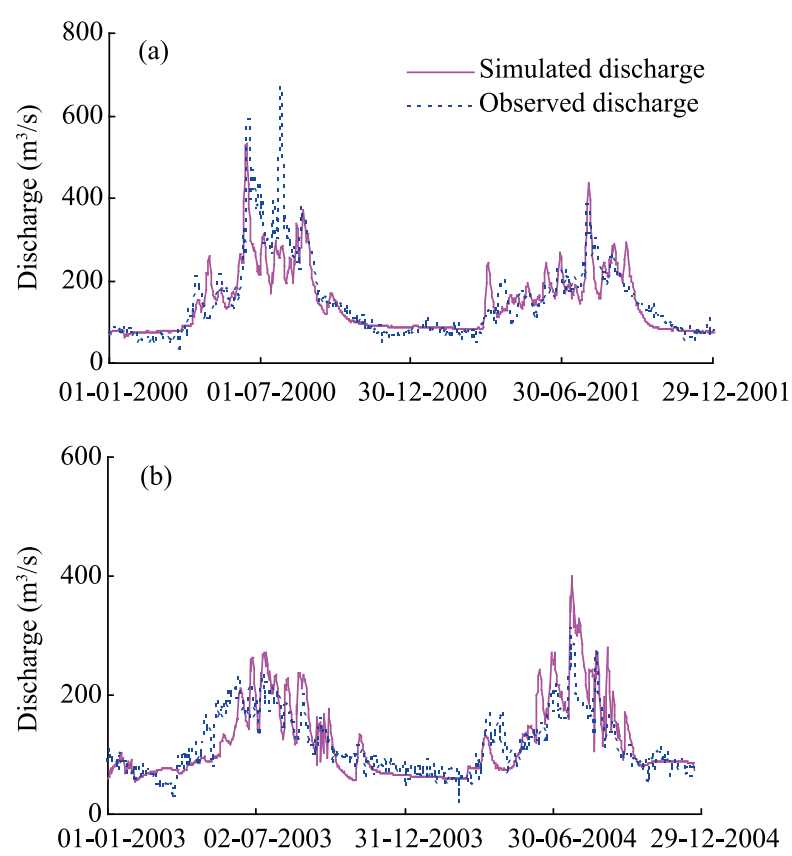

Fig. 3 Simulation results of the hydrological model; (a) calibration for DSK and (b) validation for DSK.

\section{Results and analysis}

\subsection{Solutions of the STIP}

By inputting the interval numbers of simulated stream flow and the economic data, the solutions of model (1) were obtained. Table 3 presents solutions for a basic case (scenario 1). The results indicated that solutions for the objective function value and most of the nonzero decision variables related to forestry, agriculture and ecology were intervals, while those related to municipality, industry and stockbreeding water use were deterministic values. Under the case of insufficient 
water supply, water allocation should be guaranteed first to municipality, then to stockbreeding, industry, agriculture, forestry, and then back to the ecology.

The solutions for water shortage $\left(S_{i j}^{ \pm}\right)$under given targets reflect potential system condition variations caused by uncertain inputs (as shown in Table 3). For municipality, industry and stockbreeding users, there would be zero shortage with low to high flow levels. For agriculture, the solutions of $S_{51}^{ \pm}=[599.1$, $1,040.3] \times 10^{6} \mathrm{~m}^{3}$ and $S_{52}^{t}=[0,583.5] \times 10^{6} \mathrm{~m}^{3}$ indicate that shortages may exist under low to low-medium flow levels, respectively; however, there would be no shortage under medium and high flow levels (i.e. $S_{53}^{ \pm}=$ $S_{54}^{ \pm}=S_{55}^{ \pm}=0$ ). The actual water allocation (as shown in Table 3) would be the difference between the optimized demand and the probable shortage under a given stream condition with an associated probability (i.e. $A_{i j o p t}^{ \pm}=W_{\text {iopt }}^{ \pm}-S_{i j o p t}^{ \pm} \forall i, j$ ). For example, actual water allocated to agriculture is $[1,309.9,1,752.1] \times 10^{6}$, $[1,767.7,2,351.2] \times 10^{6}, 2,351.2 \times 10^{6}, 2,351.2 \times 10^{6}$ and $2,351.2 \times 10^{6} \mathrm{~m}^{3}$ under low to high flow levels, respectively. The results indicated that water shortages would mainly occur in agriculture, ecology, and forestry sectors. Among these water users, agricultural irrigation and ecological sector consume more than $75 \%$ of the total water resources. Municipality, industry and stockbreeding bring high benefits when their water demands are satisfied and, at the same time, subject to high penalties if promised water is not delivered. Thus, in the case of insufficient water availability, allocation should be guaranteed first to municipality, then to stockbreeding, industry, agriculture, forestry, and finally to ecology. Therefore, water demands of municipality, industry and stockbreeding should be satisfied first due to their lower consumptions and allocation priorities. According to these numerical values, local managers can make different investment plans in irrigation infrastructure and farmers can conduct irrigation as planned under different flow levels. In order to evaluate the accuracy of the results, the study made a comparison between the model results and the actual allocation. For example, in 2000, the stream flow (available water resources) was $50.1 \times 10^{8} \mathrm{~m}^{3}$ and categorized as medium-high level.

Table 2 Stream flow distribution and the associated probabilities

\begin{tabular}{lccccc}
\hline Parameter & Low $(j=1)$ & Low-medium $(j=2)$ & Medium $(j=3)$ & Medium-high $(j=4)$ & High $(j=5)$ \\
\hline Flow rate $\left(q_{j}^{ \pm}, 10^{6} \mathrm{~m}^{3}\right)$ & {$[2,459,2,901]$} & {$[2,917,3,500]$} & {$[3,500,4,162]$} & {$[4,185,4,856]$} & {$[5,015,5,708]$} \\
Probability $\left(p_{j}\right)$ & 0.152 & 0.478 & 0.239 & 0.087 & 0.044 \\
\hline
\end{tabular}

Table 3 Solutions of the STIP model under scenario 1

\begin{tabular}{|c|c|c|c|c|c|c|c|}
\hline Parameter & Probability & Municipality & Industry & Stockbreeding & Forestry & Agriculture & Ecology \\
\hline Target $\left(W_{i o p t}^{ \pm}\right)\left(10^{6} \mathrm{~m}^{3}\right)$ & & 191.0 & 803.0 & 155.0 & 407.0 & $2,351.0$ & $1,051.0$ \\
\hline \multicolumn{8}{|c|}{ Shortage $\left(S_{i j \text { opt }}^{ \pm}\right)$under a flow level of $\left(10^{6} \mathrm{~m}^{3}\right)$} \\
\hline Low $(j=1)$ & 0.152 & 0 & 0 & 0 & 407.0 & {$[599.1,1,040.3]$} & $1,051.7$ \\
\hline Low-medium $(j=2)$ & 0.478 & 0 & 0 & 0 & 407.0 & {$[0,583.5]$} & $1,051.7$ \\
\hline Medium $(j=3)$ & 0.239 & 0 & 0 & 0 & {$[0,407.0]$} & 0 & {$[796.2,1,051.0]$} \\
\hline High-medium $(j=4)$ & 0.087 & 0 & 0 & 0 & 0 & 0 & {$[102.0,773.5]$} \\
\hline $\operatorname{High}(j=5)$ & 0.044 & 0 & 0 & 0 & 0 & 0 & 0 \\
\hline \multicolumn{8}{|c|}{ Allocation $\left(A_{i j}^{ \pm}\right.$opt $)$under a flow level of $\left(10^{6} \mathrm{~m}^{3}\right)$} \\
\hline Low $(j=1)$ & 0.152 & 191.0 & 803.0 & 155.0 & 0 & {$[1,309.9,1,752.1]$} & 0 \\
\hline Low-medium $(j=2)$ & 0.478 & 191.0 & 803.0 & 155.0 & 0 & {$[1,767.7,2,351.2]$} & 0 \\
\hline Medium $(j=3)$ & 0.239 & 191.0 & 803.0 & 155.0 & {$[0,407.0]$} & $2,351.0$ & {$[0,354.8]$} \\
\hline High-medium $(j=4)$ & 0.087 & 191.0 & 803.0 & 155.0 & 407.0 & $2,351.0$ & {$[277.5,949.0]$} \\
\hline $\operatorname{High}(j=5)$ & 0.044 & 191.0 & 803.0 & 155.0 & 407.0 & $2,351.0$ & $1,051.0$ \\
\hline Net benefit $\left(f^{ \pm}\right)\left(10^{6} \$\right)$ & \multicolumn{7}{|c|}{$[3,314.6,9,302.5]$} \\
\hline Penalty $\left(10^{6} \$\right)$ & \multicolumn{7}{|c|}{$[436.5,1,330.0]$} \\
\hline
\end{tabular}


Thus, the optimal water allocation pattern would be $2,335.0 \times 10^{6}, 803.0 \times 10^{6}, 407.0 \times 10^{6}, 191.0 \times 10^{6}$ and $[277.0,949.0] \times 10^{6} \mathrm{~m}^{3}$ for the agriculture, industry, forestry, municipality and ecology sectors, respectively. In comparison, the actual water allocation was $2,441.0 \times 10^{6}, \quad 672.0 \times 10^{6}, \quad 110.0 \times 10^{6}$, $176.0 \times 10^{6}$, and $61.0 \times 10^{6} \mathrm{~m}^{3}$ for the agriculture, ecology, industry, forestry and municipality sectors, respectively. The results indicated that the optimal water allocations based on the STIP model were similar to the actual water consumption for the agriculture and ecology sectors and higher than the actual water consumption for the industry, forestry and municipality sectors in 2000. Due to high stream flow in 2000, no users experienced a water shortage. The net system benefit obtained under scenario 1 would be $\$[3,314.6,9,302.5] \times 10^{6}\left(f_{o p t}^{ \pm}\right)$, which provides two extremes of the system benefit under the optimal water-allocation pattern.

\subsection{The effect of water management policies on water resources allocation}

Figure 4 shows allocation results for the six users under different scenarios corresponding to changed water resources management policies. For example, when the stream flow is low, the allocated water for the agriculture would be $[1,309.9,1,752.1] \times 10^{6}$, $[1,126.2,1,568.0] \times 10^{6}$ and $1,417.1 \times 10^{6} \mathrm{~m}^{3}$ under scenarios 1 to 3 , respectively; when the stream flow is medium, the allocated water would be $2,351.2 \times 10^{6},[2,167.0,2351.2] \times 10^{6}$ and $[1,855.8$, $2,351.2] \times 10^{6} \mathrm{~m}^{3}$, respectively; when the stream flow is high, the allocated water would be $2,351.2 \times 10^{6} \mathrm{~m}^{3}$ under all the three scenarios. For the ecology sector, when the stream flow is from low to low-medium, allocated water would be 0 , $100.0 \times 10^{6}$ and $267.0 \times 10^{6} \mathrm{~m}^{3}$ under scenarios 1 to 3 , respectively. When the stream flow is medium, the allocated water would be $[0,255.0] \times 10^{6},[100.0$, $271.8] \times 10^{6}$ and $[267.0,271.8] \times 10^{6} \mathrm{~m}^{3}$ under scenarios 1 to 3 , respectively; when the stream flow is high, the allocated water would be $1,051.0 \times 10^{6} \mathrm{~m}^{3}$ under all the three scenarios. The results indicated that interactions exist among water supplies for multiple competing users when deficit occurs. Under scenario 1, the water allocated to ecology would be decreased first in the case of insufficient water (due to its lowest benefit). After water allocated to ecology was decreased to zero, the shortage would then occur in agriculture. Under scenario 2 that promises some priority to the ecology, its minimum water demand for their user would be satisfied first, and then water allocated would be decreased in the case of insufficient water; the shortage would then occur in agriculture. Under scenario 3, around $60 \%$ of the water demand from the ecology sector should be satisfied first, then water allocated to agriculture and forestry sectors would be decreased in the case of insufficient water. Water shortages would be intensified due to ecological priorities considered under scenarios 2 and 3 .

The solutions obtained can provide an effective evaluation on pre-regulated water policies in association with economic implications (e.g. losses or penalties caused by improper policies). Figure 5 presents the expected net system benefits under different water management policies. For example, system benefit would be $\$[3,348.8,9,301.2] \times 10^{6}$ under scenario 1 ; however, when the ecological water division was considered under scenarios 2 and 3, the system benefit would be $\$[3,304.7,9,271.4] \times 10^{6}$ and $\$[1,527.7$, $9,150.3] \times 10^{6}$, respectively. The results indicated that lower- and upper-bound system benefits (of scenarios 2 and 3) were less than those of scenario 1.

\section{Conclusions}

A simulation-based two-stage interval-stochastic programming (STIP) model has been developed for water resources planning and management. The STIP was applied to a real case of water resources allocation in the Kaidu-Konqi watershed, China. Three scenarios with different water resources management policies were analyzed. The results indicated that water shortage mainly occurs in the agriculture, ecology and forestry sectors. Among these water users, the agricultural irrigation and ecological allocation occupied more than $75 \%$ of the total water consumption. Water demands from the municipality, industry and stockbreeding sectors can be satisfied due to their lower consumptions and higher economic priorities. In addition, different 

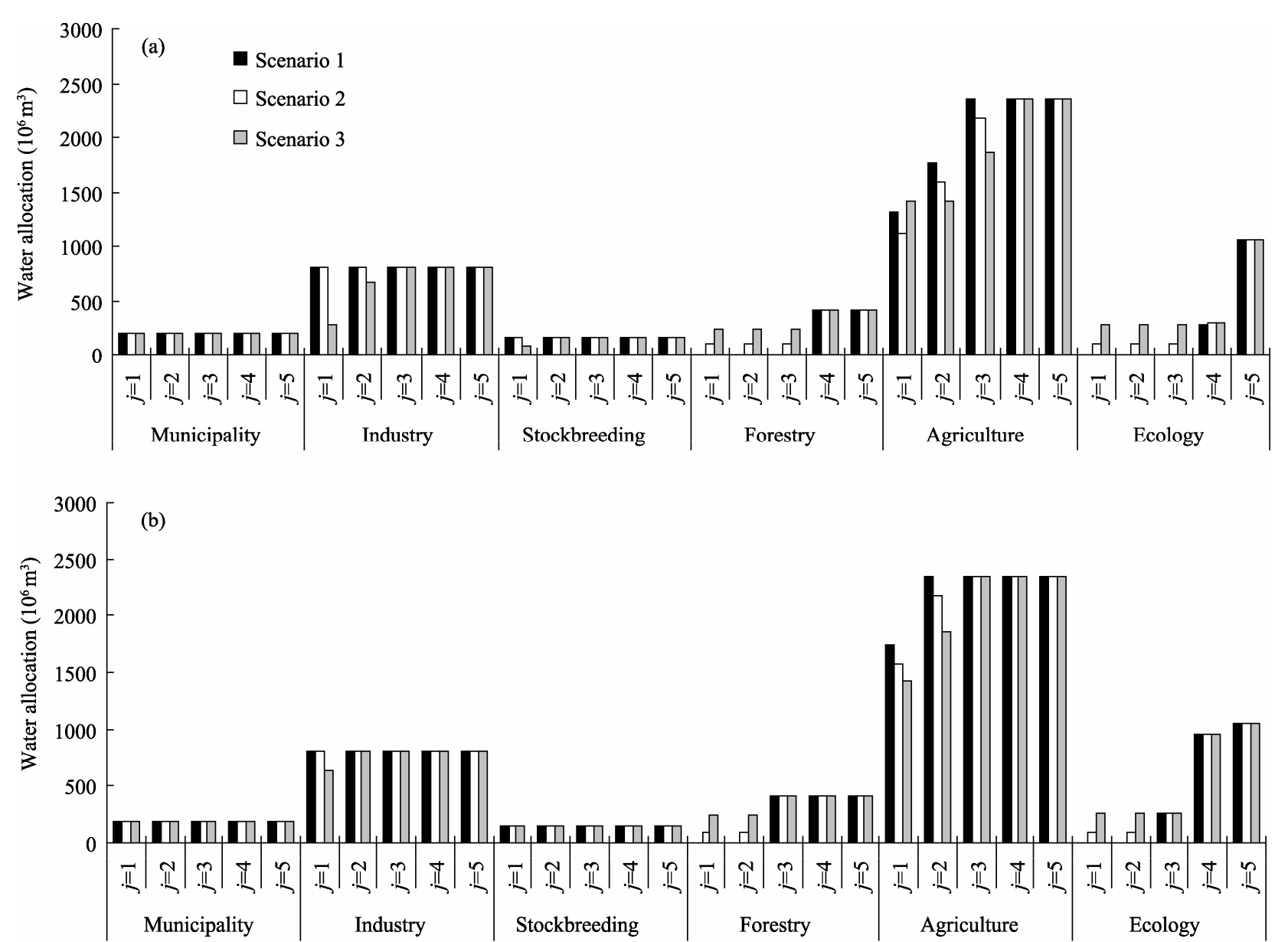

Fig. 4 Water allocation patterns under scenarios 1 to 3; (a) lower-bound and (b) upper-bound.

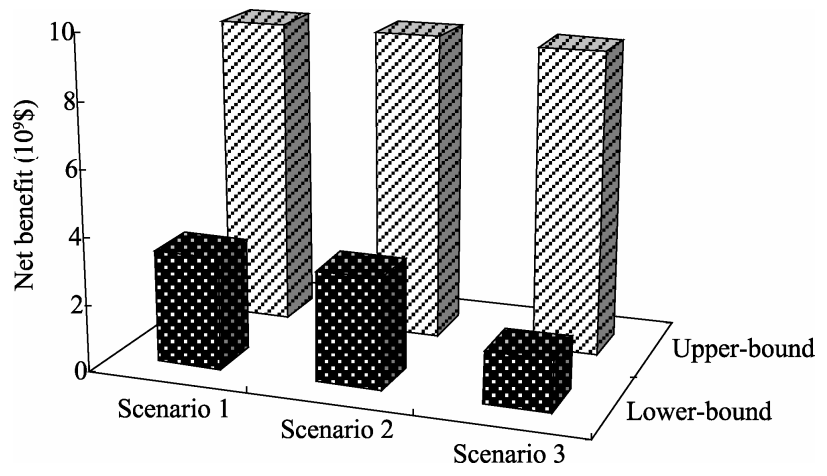

Fig. 5 Net system benefit under scenarios 1 to 3

ecological water allocation policies could result in varied system benefits.However, one main setback of this method is that it can hardly reflect the dynamic variations of system conditions, particularly when se-

\section{References}

Abbott M B, Bathurst J C, Cunge J A, et al. 1986. An introduction to the European hydrological system-Systeme Hydrologique Eu- quential interactions exist under a multistage context. Consequently, future research work shall focus on developing a multistage stochastic programming method to extend the developed STIP by permitting revised decisions in each stage based on sequentially realizations for uncertain events.

\section{Acknowledgements}

This research was supported by the National Basic Research Program of China (2010CB951002), the Dr. Western-funded Project of Chinese Academy of Science (XBBS201010 and XBBS201005), the National Natural Sciences Foundation of China (51190095), and the Open Research Fund Program of State Key Laboratory of Hydro-science and Engineering (sklhse-2012-A$03)$. The authors are grateful to the editors and anonymous reviewers for their insightful comments and suggestions.

ropeen (SHE), 2: Structure of a physically-based, distributed modeling system. Journal of Hydrology, 87: 61-77. 
Bronster A, Jaegr A, Ciintner A, et al. 2000. Integrated modeling of water availability and water use in the semi-arid northeast of Brazil. Physics and Chemistry of the Earth, 25: 227-232.

Danish Hydraulic Institute. 2007. MIKE 11 User's and Reference Manual, Horsholm: DHI Water \& Environment, Denmark.

Huang G H, Loucks D P. 2000. An inexact two-stage stochastic programming model for water resources management under uncertainty. Civil Engineering Environment System, 17: 95-118.

Huang G H, Cao M F. 2011. Analysis of solution methods for interval linear programming. Journal of Environmental Informatics, 17(2): 54-64.

Huang Y, Chen X, Li Y P, et al. 2010a. Integrated modeling system for water resources management of Tarim River Basin. Environmental Engineering Science, 27(3): 255-269.

Huang Y, Chen X, Li Y P, et al. 2010b. A fuzzy-based simulation method for modelling hydrological processes under uncertainty. Hydrological Processes, 24(25): 3718-3732.

Jing L, Chen B. 2011. Field investigation and hydrological modelling of a subarctic wetland: the deer river watershed. Journal of Environmental Informatics, 17(1): 36-45.

Li Y P, Huang G H, Nie S L. 2006. An interval-parameter multistage stochastic programming model for water resources management under uncertainty. Advances in Water Resources, 29(5): 776-789.

Li Y P, Huang G H, Nie S L, et al. 2008. Inexact multistage stochastic integer programming for water resources management under uncertainty. Journal of Environmental Management, 88: 93-107.

Li W, Li Y P, Li C H, et al. 2010. An inexact two-stage water management model for planning agriculture irrigation under uncertainty. Agriculture Water Management, 97: 1905-1914.

Luo B, Li J B, Huang G H, et al. 2006. A simulation-based interval two-stage stochastic model for agricultural nonpoint source pollu- tion control through land retirement. Science of the Total Environment, 361: 38-56.

Maqsood I, Huang G H, Huang Y F, et al. 2005. ITOM: an interval-parameter two-stage optimization model for stochastic planning of water resources systems. Stochastic Environment Research Risk Assessment, 19: 125-133.

Nash J E, Sutcliffe J V. 1970. River flow forecasting through conceptual models part I: a discussion of principles. Journal of Hydrology, 10(3): 282-290.

Paturel J E, Barrau C, Mahé G, et al. 2007. Modelling the impact of climatic variability on water resources in West and Central Africa from a non-calibrated hydrological model. Hydrological Sciences Journal, 52(1): 38-48.

Qin X S, Huang G H, Yu H. 2009. Enhancing remediation of LNAPL recovery through a response-surface-based optimization approach. Journal of Environmental Engineering, 135(10): 999-1008.

Statistical Bureau of Bayangol Mongol Autonomous Prefecture. 2000-2009. Statistical Yearbook of Bayangol. Beijing: China Statistic Press.

Wang J, Yang H, Li L, et al. 2011. The coupled routing and excess storage (CREST) distributed hydrological model. Hydrological Sciences Journal, 56(1): 84-98.

Xu Y, Huang G H, Qin X S. 2009. An inexact two-stage stochastic robust optimization model for water resources management under uncertainty. Environmental Engineering Science, 26(12): 1765-1767.

Yang Y H, Chen Y N, Li W H, et al. 2010. Impacts of climatic change on river runoff in Northern Xinjiang of China over last fifty years. Chinese Geographical Science, 20(3): 193-201.

Zhang Y C. 2006. A study on hydrological process response to environmental change in Kaidu Watershed. Ph.D Dissertation, Beijing: Chinese Academy of Sciences. 